\title{
Global mild solution for the Navier-Stokes-Nernst-Planck-Poisson system in Besov-weak-Herz spaces
}

\author{
Aibo Liu ${ }^{1}$ and Jianing $\mathrm{Xie}^{2}$ \\ ${ }^{1}$ Liaoning Normal University \\ ${ }^{2}$ Dongbei University of Finance and Economics
}

May 29, 2020

\begin{abstract}
We study a coupled Navier-Stokes-Nernst-Planck-Poisson system arising from electrohydrodynamics in critical Besov-weakHerz spaces. When the initial value sufficiently small, we prove the existence and uniqueness of global mild solution to the cauchy problem in this spaces for $\$ \mathrm{n} \backslash$ geq $3 \$$. The spaces is larger than some other known critical spaces.
\end{abstract}

\section{Hosted file}

Global mild solution for the Navier--Stokes--Nernst--Planck--Poisson system in Besov-weak-Herz spaces.p available at https://authorea.com/users/327909/articles/455300-global-mild-solution-for-thenavier-stokes-nernst-planck-poisson-system-in-besov-weak-herz-spaces 